\title{
Dinámica de cambios en el agroecosistema de cafetal bajo sombra en la cuenca alta de La Antigua, Veracruz
}

\section{Dynamics of changes in the agroecosystem of a shade-grown coffee plantation in the upper La Antigua river basin, Veracruz}

\author{
Marco Antonio Espinoza-Guzmán', Lázaro Rafael Sánchez Velásquez², María del Rosario Pineda López², \\ Francisco Javier Sahagún Sánchez ${ }^{3^{*}}$, David Aragones Borrego ${ }^{4}$ y Zemei Fernando Reyes Garcíal
}

\footnotetext{
1 'Universidad Veracruzana. Facultad de Biología. Xalapa, Veracruz, México. marco_spinoza@yahoo. com.mx; zemeirega@gmail.com

2 Universidad Veracruzana. Instituto de Biotecnología y Ecología Aplicada. Xalapa, Veracruz, México. lasanchez@uv.mx; rosariorosariop@amail.com
}

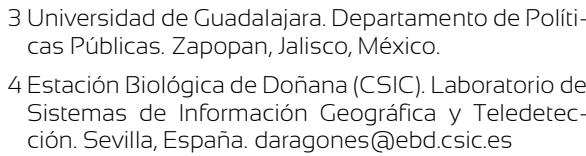

* Autor de correspondencia.

francisco.sahagun@cucea.udg.mx

\section{RESUMEN}

En México, los cafetales bajo sombra constituyen parte del paisaje agroforestal y representan el refugio de una alta diversidad biológica. Sin embargo, están sujetos a procesos de transformación derivados de las necesidades de los productores y las demandas del mercado, lo que afecta la configuración del agroecosistema. Este trabajo tuvo como objetivo cuantificar las transformaciones de los usos de suelo relacionados con los cafetales en la cuenca alta de La Antigua, durante un periodo de 24 años. Se analizó la dinámica de cambio en las coberturas de uso del suelo y vegetación, mediante la generación de cartografía con la técnica de clasificación supervisada de las imágenes LANDSAT (TM, ETM y OLI) de los años de 1993, 2003 y 2017 y se analizaron los catalizadores de los cambios que determinan la configuración actual del paisaje agroforestal con base en entrevistas a actores clave de la región. Los resultados muestran que la transformación de los sistemas agroforestales de los cafetales bajo sombra se debe a los cambios de uso del suelo y los programas gubernamentales implementados en la zona. La cafeticultura representa una de las principales fuentes de ingreso en la región del Corredor Ecológico de la Sierra Madre Oriental del estado de Veracruz, por lo que se requiere el desarrollo de estrategias que garanticen el mantenimiento y la sostenibilidad de esta actividad económica en la zona para evitar los impactos potenciales al desarrollo local de las comunidades, así como la afectación o pérdida de los ecosistemas naturales remanentes en la región.

PALABRAS CLAVE: cambios de uso del suelo y vegetación; clasificación supervisada; sistemas agroforestales; Sierra Madre Oriental; teledetección.

\section{ABSTRACT}

In Mexico, shade-grown coffee agroecosystems form part of the agroforestry landscape and provide shelter for a wide range of biological diversity. However, they are subject to transformation processes derived from the needs of producers and market demands, which affects the configuration of the agroecosystem. The present study aimed to quantify the transformations of land use cover related to coffee plantations in the upper La Antigua river basin, that has occurred over a period of 24 years. The dynamics of change in the coverage of land use and vegetation was analyzed, by generating cartography with the supervised classification technique of the LANDSAT images (TM, ETM, and OLI) of the years of 1993, 2003 and 2017 and the analysis of the factors that have promoted the changes that determine the current configuration of the agroforestry landscape, based on interviews with key stakeholders in the region. The results show that the transformation of the agroforestry systems in shade-grown coffee plantations is caused by land use changes and the government programs implemented in the region. Coffee growing represents one of the main sources of income in the Ecological Corridor of the Sierra Madre Oriental of the state of Veracruz, which requires the development of strategies that guarantee the maintenance and sustainability of this economic activity in the zone to avoid the potential impacts to the local development of the communities, as well as the affectation or loss of the natural ecosystems remaining in the region.

KEYWORDS: land use cover change; supervised classification; agroforestry systems; Sierra Madre Oriental; remote sensing. 


\section{INTRODUCCIÓN}

Los procesos de cambio en el uso del suelo constituyen un tema de interés prioritario en la agenda ambiental global debido a la influencia que tienen sobre la pérdida de biodiversidad y el cambio climático (Lambin, Geist y Lepers, 2003; Turner, Lambin y Reenberg, 2007). Entre los impactos más importantes se destacan los cambios en la cubierta vegetal original y su sustitución por tierras dedicadas a usos agrícolas y de pastoreo (Lambin y Meyfroidt, 2010). Especialmente en los últimos 50 años, se ha observado como la cobertura vegetal ha tenido modificaciones en su estado, estructura, composición y extensión, lo que repercute no sólo en las condiciones del ambiente y los recursos disponibles, sino también en los aspectos socioeconómicos. Las modificaciones en los ecosistemas son el resultado de diversas interacciones complejas entre factores físicos, biológicos, económicos, políticos y sociales (Mas, 1999; Mas y Fernández, 1999; Lambin et al., 2001; Archer, Dawson, Kreuter, Hendrickson y Halloran, 2008; Fonseca-Cepeda, Idrobo y Restrepo, 2019). Aun cuando estos cambios son de tendencia global, estos han sido más evidentes en las regiones tropicales (Foley et al., 2005).

Al haber una gran cantidad de factores que generan cambios de uso del suelo y coberturas de vegetación (Alexander et al., 2015), se requiere un enfoque de estudio espacio-temporal que permita entender la dinámica de los procesos (Geist y Lambin, 2001). Existe una gran cantidad de estudios basados en imágenes de satélites para evaluar los cambios de uso del suelo (Verburg, Paul, Schot, Dijst y Veldkamp, 2004), y se han utilizado métodos cualitativos y cuantitativos para evaluar la influencia de las variables socioeconómicas sobre estos procesos (Chomitz y Gray, 1996; Agarwal, Green, Grove, Evans y Schweik, 2002)

Las áreas cafetaleras bajo sombra han sido reconocidas por su importancia en la provisión de servicios ecosistémicos y la conservación de la diversidad biológica (Perfecto y Vandeermer, 2010). Estudios realizados en estos sistemas de producción señalan que los servicios ambientales provistos por estos agroecosistemas se incrementan conforme es mayor su diversidad y la complejidad estructural (Moguel y Toledo, 1999). En México más de $99 \%$ de los predios cafetaleros se encuentra bajo cobertura arbórea (Moguel y Toledo, 1999; Fideicomiso Instituidos en Relación con la Agricultura [FIRA], 2016), esto representa más de 600 mil hectáreas, donde los cafetaleros están generando ingresos por la producción de café y de forma directa e indirecta apoyan la conservación de la diversidad biológica y garantizan el suministro de servicios ecosistémicos. Diversos autores reconocen que los agroecosistemas de café ayudan al mantenimiento de la diversidad debido a su complejidad estructural y a que disminuyen la presión sobre las áreas remanentes de vegetación original (Redo et al., 2009); por ejemplo, aquellos que sirven como zona de amortiguamiento al bosque mesófilo de montaña (Williams-Linera, Manson e Isunza-Vera, 2002; CastilloCapitán, Ávila-Bello, López-Mata y León, 2014). Sin embargo, las áreas cafetaleras son susceptibles a ser modificadas por cambios de uso del suelo cuando los productores enfrentan crisis económicas por largos periodos con precios bajos o problemas fitosanitarios que afectan el rendimiento (Ponette-González, 2007), lo que propicia el cambio en la vocación de las tierras a usos agropecuarios con las consecuencias previsibles para la provisión de servicios ecosistémicos (Castillo-Capitán, et al. 2014).

La descripción de los cambios de uso del suelo en zonas productoras de café ha sido globalmente documentada en una vasta literatura, pero muy comúnmente en áreas marginales y alejadas de los centros urbanos demandantes de terrenos para su expansión, por ejemplo, Ambinakudige y Choi (2009) en la India, Bass (2006) en Honduras, Schmitt-Harsh (2013) en Guatemala. Por otra parte, se han utilizado modelos PROBIT (probabilidad + unit (unidad)) ordenados en áreas de café en Costa Rica (Bosselmann, 2012), donde se ha concluido que cuando el número de miembros de la familia aumenta y estos se dedican a otras actividades, es cuando más frecuentemente los cafetales son sustituidos por otros usos de suelo con menor cobertura (potreros y caña de azúcar). 
En México, Ellis, Baerenklau, Marcos-Martínez y Chávez (2010) observaron que, en áreas marginales de producción de café en el norte de Veracruz, ubicadas entre los $300 \mathrm{~m}$ y los $800 \mathrm{~m}$ snm y donde la producción es menor y de baja calidad (Castillo-Capitán, et al. 2014), los productores han cambiado sus cafetales por pastizales para ganado, principalmente cuando estos se encuentran en terrenos con poca o nula pendiente. Con base en lo anterior surge la siguiente pregunta: ¿Cuáles son los factores que han promovido los cambios de uso de suelo en las áreas cafetaleras en la cuenca alta de La Antigua, Veracruz?

El presente estudio se desarrolló en los municipios veracruzanos que conforman la parte alta de la Cuenca de La Antigua, región reconocida por la buena calidad del café (FIRA, 2016), la presencia de bosque mesófilo de montaña y por estar incluida en el Corredor Ecológico de la Sierra Madre Oriental (Cesmo) (Piñar-Álvarez, EspinozaGuzmán, Jiménez-Olivencia y Loreto-Bermúdez, 2016). Esta parte de la cuenca se caracteriza también por su proximidad a centros urbanos como la ciudad de Xalapa (capital del estado de Veracruz) y Coatepec, región que tiene un vigoroso incremento de población humana desde la década de los años noventa.

\section{OBJETIVOS}

El estudio tuvo dos objetivos: 1) Ubicar y cuantificar los cambios de uso del suelo y los sistemas agroforestales cafetaleros bajo sombra en la parte alta de la Cuenca de la Antigua en el período de 1993 a 2017, y 2) Realizar una evaluación cualitativa de la dinámica de los procesos de cambio de los sistemas agroforestales cafetaleros bajo sombra en la región estudiada.

\section{MATERIALES Y MÉTODOS}

\section{Área de estudio}

La cuenca de La Antigua está ubicada en la zona centro del estado de Veracruz en la vertiente oriental del sistema volcánico de Cofre de Perote y Pico de Orizaba, perteneciente al Cinturón Neovolcánico Transversal Mexicano. Colinda al norte con la cuenca del río Actopan y al sur con las cuencas del Atoyac y Jamapa-Cotaxtla. La cuenca en cuestión, por su posición en ladera oriente del Cofre de Perote (García, Gallardo, Nava, Ruiz y Escamilla, 2006), está expuesta a vientos alisios, los cuales están cargados de humedad y chocan con la estructura volcánica para luego ascender hasta condensarse, estas características topográficas generan diversas condiciones climáticas y biológicas. Debido a esto, se presenta un mosaico de paisajes en los que se pueden identificar: páramo de altura, bosque de coníferas, bosque mesófilo de montaña y bosque tropical caducifolio, entre otros (Muñoz-Villers y LópezBlanco, 2007). El estudio se realizó en la porción alta de la cuenca La Antigua, que fue delimitada con base en los modelos digitales de elevación del Instituto Nacional Estadística y Geografía E14B26, E14B27, E14B36 y E14B37 (Instituto Nacional de Estadística, Geografía e Informática [Inegi], 2005) mediante el trazo de las curvas de nivel e incluye total o parcialmente los municipios de Acajete, Ayahualulco, Coatepec, Cosautlán de Carvajal, Emiliano Zapata, Ixhuacán de los Reyes, Las Vigas de Ramírez, Perote, Teocelo, Tlalnehuayocan, Tlaltetela, Xalapa y Xico, todos pertenecientes al estado de Veracruz (Inegi, 2010) (Fig. 1).

\section{Análisis espacial de los cambios en los sistemas agroforestales cafetaleros bajo sombra}

La distribución de los sistemas agroforestales cafetaleros bajo sombra (Sacbs), se delimitó con base en el procesamiento de imágenes multi-espectrales del sensor LANDSAT del path 25 y row 47 -5 TM (15/02/1993), 7 ETM (09/12/2003) y 8 OLI (27/01/2017). Se realizó una corrección geométrica y atmosférica de las imágenes anteriormente citadas conforme a los procedimientos señalados por Chuvieco (2008), y se utilizó la imagen correspondiente a 2017, como referencia para realizar ocho recorridos de campo en los que se obtuvieron 377 puntos geo referenciados sobre el terreno para crear las firmas espectrales de las imágenes LANDSAT, que permitieron generar la clasificación supervisada con 12 categorías de uso del suelo y vegetación (acahual, agrícola, Sacbs, agua, 
infraestructura, áreas sin datos por nubes y sombras, bosque mesófilo de montaña, pastizal, páramo de altura, bosque de coníferas, bosque tropical caducifolio, y sin vegetación) en la cuenca (Hoffmann, 1993; EspinozaGuzmán, 2012; Reyes, 2015; Reyes, 2018).

Para generar los mapas de uso del suelo y vegetación se utilizó el algoritmo de clasificación supervisada con el agrupamiento estadístico de máxima probabilidad (Argañaraz y Entraigas, 2011), con base en los recorridos de campo realizados entre los meses de noviembre de 2016 a enero de 2017, con la finalidad de garantizar la consistencia entre lo observado en el terreno y lo captado por el sensor. Para determinar la precisión de la clasificación se realizó un análisis geoestadístico para la identificación de las fuentes de error (Jennes y Judson, 2007); se generó una matriz de confusión y se elaboró un análisis "Kappa de Cohen”, y de esta manera se validó estadísticamente cada clasificación y se estimó la tasa de error en la clasificación general, la especificidad y la sensibilidad del modelo; además de mostrar los errores de omisión y comisión. Posteriormente se elaboraron los mapas de los usos del suelo, basados en las firmas espectrales (Chuvieco, 2008) y se generaron las clasificaciones para los años 1993 y 2003.

Para conocer el cambio de uso de los Sacbs se realizó un análisis multitemporal postclasificación, el cual consiste en la transposición de dos mapas generados de la clasificación supervisada para los periodos 1993-2003 y 2003-2017, así como durante el periodo global de 24 años del estudio (1993-2017).
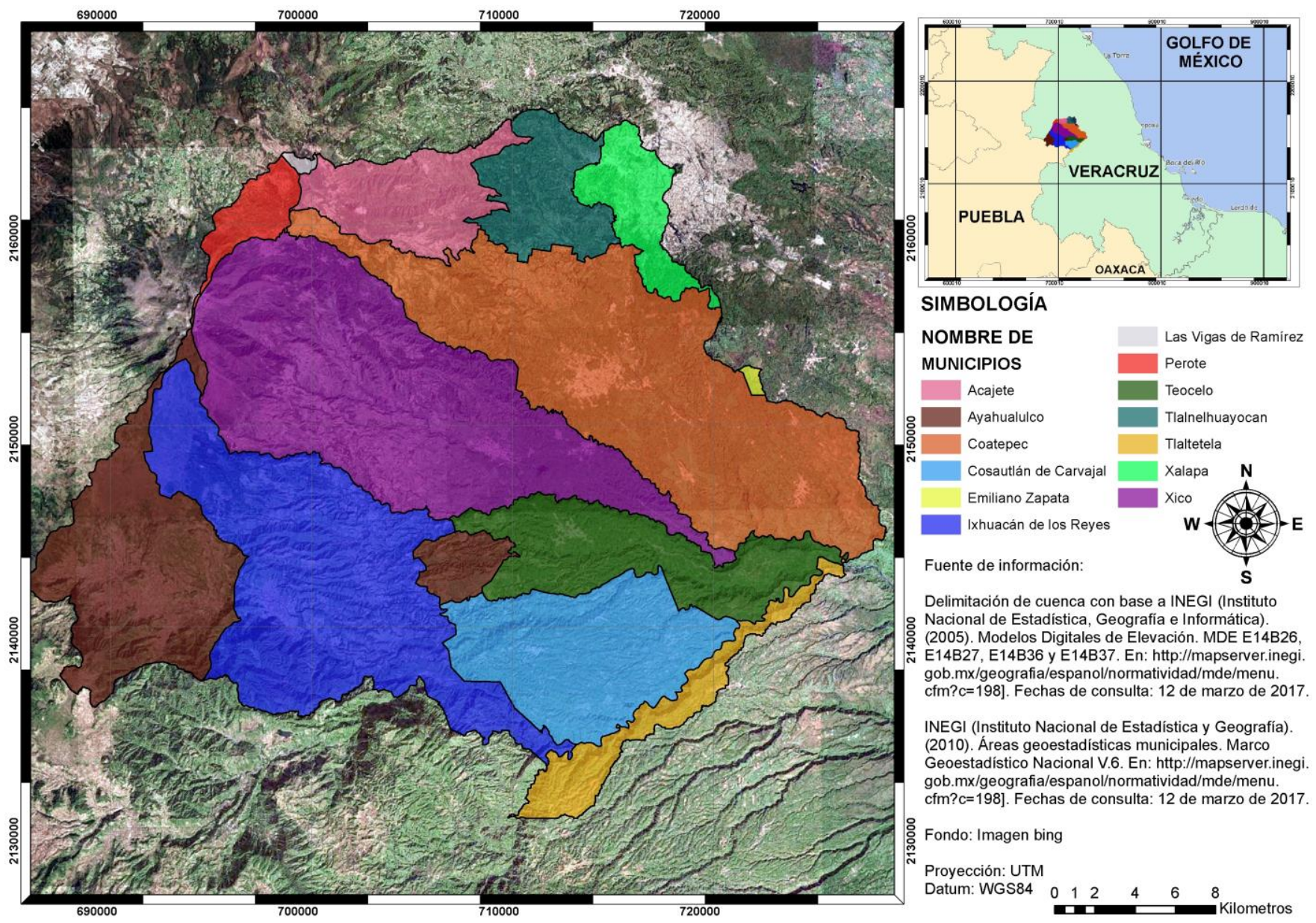

FIGURA 1. Ubicación del área de estudio en la cuenca alta de La Antigua. 
Para estimar las tasas de cambio de uso en el suelo en los periodos analizados, se usó la ecuación (1) (Velázquez et al., 2002) recomendada por la Food and Agriculture Organization [FAO] (1996) que, de acuerdo con PalacioPrieto et al. (2004), es coherente y replicable para la evaluación y análisis de la dinámica del cambio de coberturas en México, y es definida como la proporción de la superficie que cambia en su cobertura en un periodo de tiempo determinado. El conocer este dato permite deducir los efectos de la degradación de los ecosistemas, la disminución de la diversidad biológica y la consecuente pérdida de servicios ecosistémicos (Mas y Fernández, 2003).

$$
\delta_{n}=\left[\frac{S_{2}}{S_{1}}\right]^{1 / n}-1
$$

Donde:

$\delta_{n}=$ tasa de cambio (para expresar en porcentaje se multiplicó por 100)

$S_{1}=$ superficie en fecha 1

$S_{2}=$ superficie en fecha 2

$n=$ número de años entre las dos fechas

\section{Evaluación cualitativa de los cambios de uso del suelo en el área de estudio}

La información cualitativa se obtuvo mediante 20 entrevistas semiestructuradas a profundidad Mahoney (1997), realizadas a actores clave de la zona de estudio entre los meses de octubre a marzo de 2017 de acuerdo con lo propuesto por Lofland y Lofland (1995) y Touraine (1997). (Anexo 1). Los entrevistados fueron identificados tanto en los recorridos de campo, como con la ayuda de organizaciones de la sociedad civil locales y autoridades municipales de la región. Las entrevistas a profundidad fueron grabadas (previa autorización para ello), transcritas y analizadas de acuerdo con la metodología propuesta por Bardin (1996), que considera los criterios de exclusión mutua, homogeneidad, pertinencia, objetividad, fidelidad y productividad para el análisis de contenido y su posterior codificación e interpretación. Lo anterior, aportó elementos de análisis de la información desde un enfoque mixto (cuantitativo y cualitativo), que se entrelazó a lo largo de los resultados desde el punto de vista socioambiental y económico.

\section{Resultados}

De la delimitación de la parte alta de la cuenca de La Antigua resultó una superficie de 88970 ha, en la que se determinó que para el año 1993 los SACBS tuvieron presencia en 16116.29 ha (18.11\%), mientras que para el año 2003 ocupaban 6.46\% de la superficie (14 641.69 ha), por lo que tuvo una tasa de cambio de $-0.95 \%$. Para 2017 este agroecosistema ocupó 16777.30 ha (18.86\%), con una tasa de cambio de $0.98 \%$ entre el año 2003 y el año 2017. Para el periodo de 24 años (1993 a 2017) se estimó la tasa de cambio de $0.17 \%$ (Tabla 1, Fig. 2).

\section{Situación institucional relacionada con la cafeti- cultura antes de 1993}

Los entrevistados señalaron que, antes del año de 1993, se presentaron obstáculos político-administrativos para el desarrollo de los Sistemas agroforestales cafetaleros bajo sombra (Sacbs). Se destaca la desaparición, en 1992, del Instituto Mexicano del Café (Inmecafé), órgano del gobierno federal mexicano que aglutinó las actividades productivas, asesoría técnica y de comercialización para los cafeticultores mexicanos y cuyo desmantelamiento generó graves afectaciones a este sector. Esto sumado a la severidad de la "roya" del café (Hemileia vastatrix Berk \& Br.) (FIRA, 2016), que desde 1989 hasta el año 2017 se ha desarrollado en la zona de estudio, generó una situación que influyó en la reducción de los Sacbs; lo que pone en evidencia -de acuerdo con los entrevistados- la ausencia de una estrategia sólida para dar una solución integral a este problema fitosanitario.

Por otra parte, en las entrevistas también se mencionó, que a partir de 1990 se tuvo un auge la sustitución de Sacbs por cañaverales u otros cultivos como el de cítricos, lo que pudo estar relacionado con la llegada a la región de habitantes del centro de la República Mexicana que se asentaron principalmente en las proximidades de Coatepec, 
Xalapa y Xico, luego los sismos de 1985 ocurridos en el Distrito Federal (actualmente Ciudad de México). Esta situación generó un incremento en las tasas de cambio de uso del suelo por intereses inmobiliarios que se presentaron de manera relevante en la zona. Dentro de las repercusiones sociales desde la desaparición del Inmecafé, destaca la baja productividad de los Sacbs, que enfatizó el fenómeno migratorio nacional e internacional, tanto de finqueros como de trabajadores de los sistemas agroforestales.

Situación y cambios en los sistemas agroforestales cafetaleros bajo sombra en el periodo de 1993 a 2003

De 1993 a 2003, 20.83\% de la superficie de Sacbs cambio a otros usos de suelo (Tabla 1), 14.24\% pasó a vegetación secundaria (acahual) y 3.05\% cambio a bosques mesófilos tendentes a la madurez (ambos ecosistemas se formaron por los cafetales abandonados), mientras que 2.20\% fue convertido en campos agrícolas (cultivos de caña de azúcar principalmente); $0.85 \%$, que equivale a 194.01 ha se convirtieron en calles, carreteras y casas habitación. De la misma manera se observa que en este periodo se mantuvieron como Sacbs 11353.36 ha (49.65\%) y fueron convertidas a este agroecosistema 3288.33 ha (14.38\%), proveniente principalmente de acahuales que fueron rehabilitados $(5.58 \%)$, bosque mesófilo de montaña $(4.40 \%)$ y en la porción baja de la cuenca sobre la cota de los $1000 \mathrm{~m}$ snm el bosque tropical caducifolio (3.38\%). En este periodo la superficie total de los Sacbs fue de 16116.29 ha (Tabla 1; Fig. 2).

TABLA 1. Superficie en hectáreas, porcentaje correspondiente al total de la cuenca y tasa de cambio de los sistemas agroforestales cafetaleros bajo sombra por año.

\begin{tabular}{|c|c|c|c|c|c|c|}
\hline \multirow{3}{*}{ Tasa de cambio de los Sacbs } & \multicolumn{2}{|c|}{ Periodo 1993 a 2003} & \multicolumn{2}{|c|}{ Periodo 2003 a 2017} & \multicolumn{2}{|c|}{ Periodo 1993 al 2017} \\
\hline & \multicolumn{2}{|c|}{-0.95} & \multicolumn{2}{|c|}{0.98} & \multicolumn{2}{|c|}{0.17} \\
\hline & hectáreas & porcentaje & hectáreas & porcentaje & hectáreas & porcentaje \\
\hline $\begin{array}{l}\text { Superficie de Sacbs en la cuenca alta } \\
\text { de La Antigua, Veracruz. }\end{array}$ & 16116.29 & 18.11 & 14641.69 & 16.46 & 16777.30 & 18.86 \\
\hline Dejaron de ser Sacbs & 4762.93 & 20.83 & 3342.35 & 14.62 & 4920.12 & 21.52 \\
\hline Sacbs a acahual & 3255.19 & 14.24 & 1688.90 & 7.39 & 2354.86 & 10.30 \\
\hline Sacbs a agrícola & 503.33 & 2.20 & 502.59 & 2.20 & 852.94 & 3.73 \\
\hline Sacbs a infraestructura & 194.01 & 0.85 & 440.68 & 1.93 & 831.57 & 3.64 \\
\hline Sacbs a bosque mesófilo de montaña & 696.92 & 3.05 & 430.65 & 1.88 & 628.32 & 2.75 \\
\hline Sacbs a pastizal & 15.82 & 0.07 & 82.33 & 0.36 & 131.96 & 0.58 \\
\hline Sacbs a bosque tropical caducifolio & 97.04 & 0.42 & 187.94 & 0.82 & 108.86 & 0.48 \\
\hline Sacbs a sin vegetación & 0.61 & 0.00 & 1.15 & 0.01 & 2.92 & 0.01 \\
\hline Sacbs a agua & 0.00 & 0.00 & 8.09 & 0.04 & 8.71 & 0.04 \\
\hline Se mantuvo como Sacbs & 11353.36 & 49.65 & 11299.35 & 49.42 & 11196.17 & 48.97 \\
\hline Se incorporó a la categoría Sacbs & 3288.33 & 14.38 & 5479.14 & 23.96 & 5581.17 & 24.41 \\
\hline Acahual a Sacbs & 1275.39 & 5.58 & 3838.56 & 16.79 & 2547.28 & 11.14 \\
\hline Agrícola a Sacbs & 230.96 & 1.01 & 223.14 & 0.98 & 336.49 & 1.47 \\
\hline Bosque mesófilo de montaña a Sacbs & 1006.27 & 4.40 & 1003.31 & 4.39 & 1612.04 & 7.05 \\
\hline Pastizal a Sacbs & 3.49 & 0.02 & 50.08 & 0.22 & 27.22 & 0.12 \\
\hline Bosque tropical caducifolio a Sacbs & 771.81 & 3.38 & 362.90 & 1.59 & 1056.78 & 4.62 \\
\hline Sin vegetación a Sacbs & 0.41 & 0.00 & 1.15 & 0.01 & 1.36 & 0.01 \\
\hline
\end{tabular}


a)

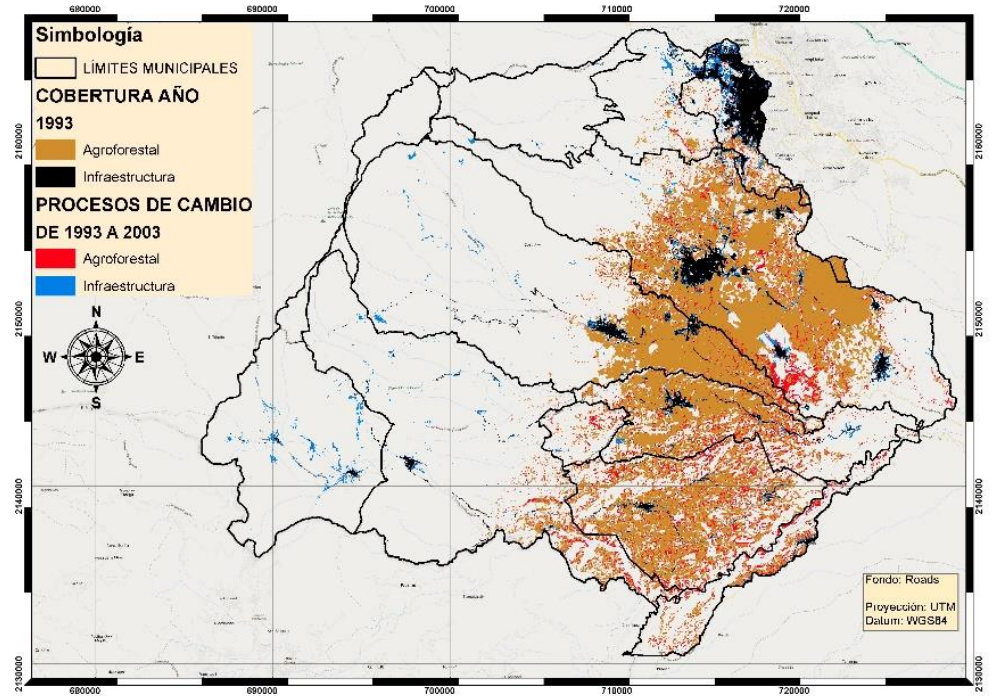

b)

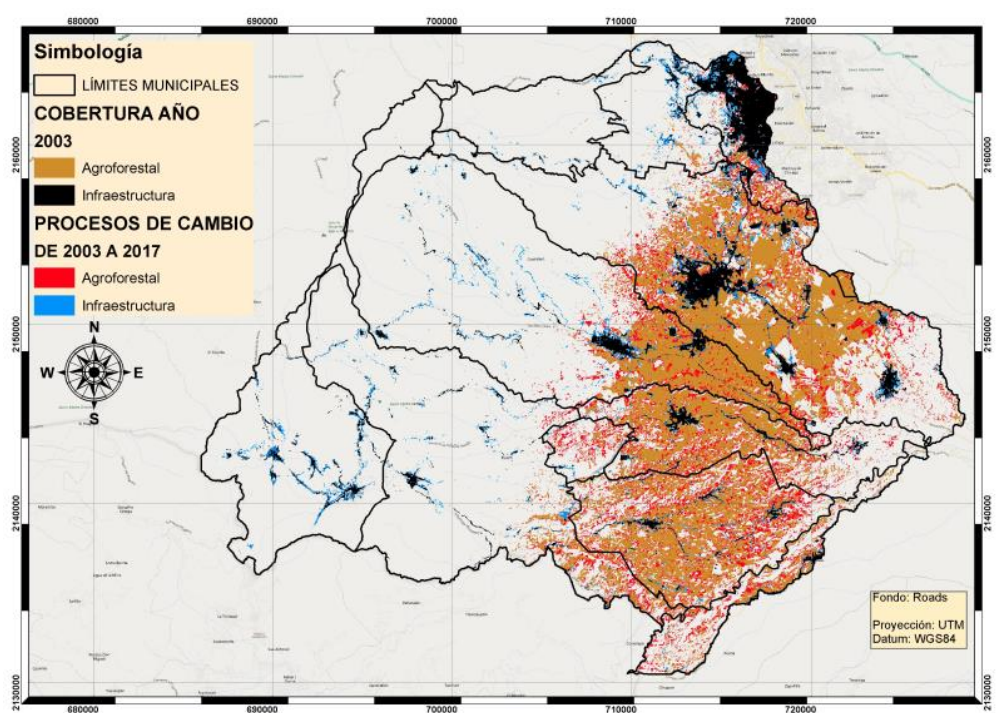

c)

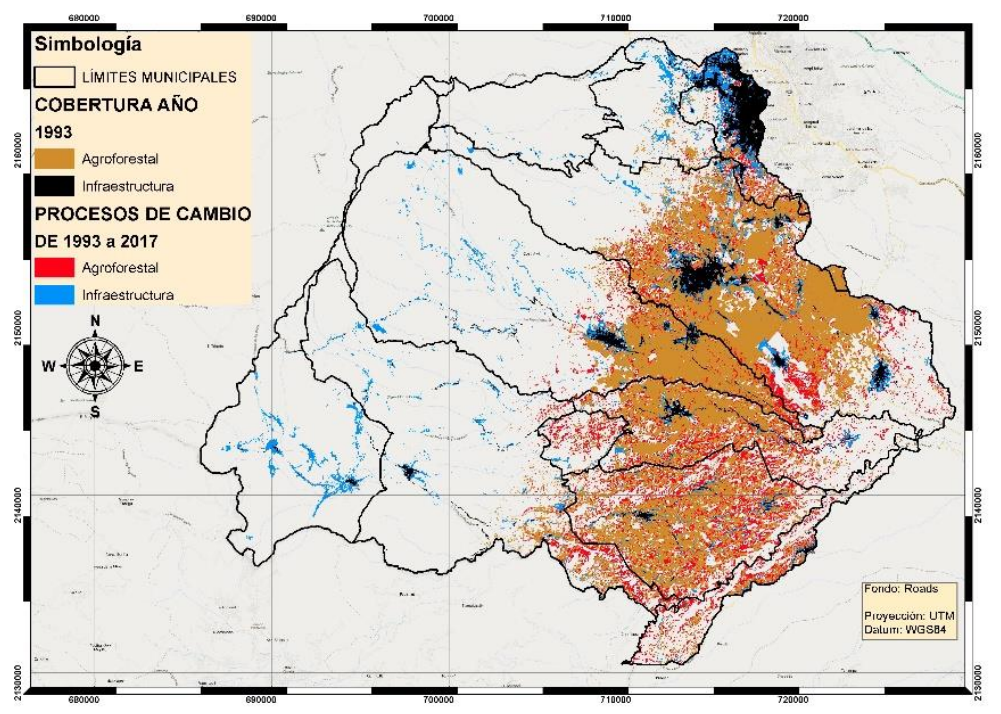

FIGURA 2. Cambios en el uso de suelo del área de estudio en los periodos a) 1993 2003, b) 2007 - 2017 y c) 1993 - 2017. 
Entre 1993-1999, los productores trabajaron con los Sacbs que quedaron y recuperaron algunas de las fincas cafetaleras que fueron abandonadas desde el año de 1993. Por otra parte, iniciaron en la región apoyos gubernamentales de Procampo (Programa de Apoyos Directos al Campo) y Pronare (Programa Nacional de Reforestación), que inciden en los Sacbs, al ser incentivados con plantas para la cubierta forestal de los cafetales. Otros apoyos a los sistemas agroforestales fueron a través del Consejo Mexicano del Café y Alianza para el Campo ambos en el año de 1994, con financiamiento a fondo perdido, impulsaron el mejoramiento de las plantaciones cafetaleras. Por otra parte, en el año de 1998 el sector cafetalero afrontó otro momento de crisis relacionada con la caída de los precios del café en el mercado internacional (Organización Internacional del Café [OIC], 2011), situación desfavorable en regiones productoras en México incluyendo la zona de estudio.

\section{Situación y cambios en los SACBS en el periodo de 2003 a 2017}

En el periodo de 2003 a 2017, 7.39\% (1688.9 ha) de los Sacbs se convirtieron en acahuales; $1.88 \%$, (430.65), se sumaron al bosque mesófilo de montaña y $0.82 \%$, (187.94 ha) a bosque tropical caducifolio. En conjunto representan $10.09 \%$ (2307.49 ha) de la superficie de cafetales que fueron abandonados en este periodo. Por otra parte, fueron convertidos a campos agrícolas $2.20 \%$ (502.59 ha) y a infraestructura 1.93\% (440.68 ha) (Tabla 1, Fig. 2).

En este periodo, la superficie que se mantuvo como Sacbs fue 11299.35 ha (49.42\%), y se sumaron a este agroecosistema 5479.14 ha $(23.96 \%)$ provenientes de coberturas de acahuales (3838.56 ha, 16.79\%), de bosque mesófilo de montaña (1003.31 ha, 4.39\%), de bosque tropical caducifolio con 362.90 ha $(1.59 \%)$ y de superficie agrícola 223.14 ha $(0.98 \%)$. La superficie total de los Sacbs para este periodo fue de 14641.69 ha (Tabla 1, Fig. 2).

Derivado del análisis de contenido (entrevistas a profundidad), destacan factores que afectan negativamente a los sistemas agroforestales, que llevaron a la caída en su producción del café como fue la aparición de la broca del café en el año de 1999, situación que mermó la producción y calidad. Sumando a la situación anterior se agrega la eliminación de los apoyos tanto de Alianza para el Campo (año 2000), como la del Consejo Mexicano del Café (desaparición de esta institución en el año 2004), lo que propició el abandono de fincas cafetaleras.

Por otro lado, los entrevistados manifestaron que, derivado de la recuperación de algunas de las fincas abandonadas, en años posteriores se registró un aumento en la superficie de los sistemas agroforestales. En cuanto a los apoyos recibidos en el sector continuó la dotación de plantas para la sombra de café por parte de Pronare y Procampo (que durante el año de 1993 incidió en la región), además de la entrega de pesetilla (plántula de café) para mitigar pérdida de las plantas por plagas, aunque con mayor importancia en este periodo, aunque a decir de los entrevistados se observa desde el año de 1998.

\section{Situación de los SACBS en el periodo de 1993 a 2017}

En este periodo los Sacbs crecieron de 16116.29 ha en el año de 1993 a 16777.30 ha para el año de 2017, lo que generó una tasa de incremento positivo de $0.17 \%$ (Tabla 1 , Fig. 2), manteniéndose en esta categoría durante este periodo 48.97\% (11 $196.17 \mathrm{ha}$ ), superficie que proviene principalmente de acahuales $11.14 \%$ (2547.28 ha), bosque mesófilo de montaña 7.05\% (1612.04 ha) y bosque tropical caducifolio 4.62\% (1056.78 ha). Mientras que en este mismo periodo la transformación de los Sacbs a otros usos y tipos de vegetación fueron hacia acahuales 10.30\% (2354.86 ha), a uso agrícola 3.73\% (852.94 ha) y en infraestructura 3.64\% (831.57 ha), principalmente (Tabla 1).

\section{DISCUSIÓN}

El análisis de 24 años referentes al cambio de cobertura y uso del suelo de la porción alta de la cuenca del río La Antigua en el estado de Veracruz, muestra una realidad dinámica que coincide con lo señalado por Mas (1999); Mas y Fernández (1999) y Lambin et al. (2001) para otras zonas, donde existe influencia de procesos de transformación 
socioeconómicos y políticos, que definen los diferentes paisajes tanto naturales como artificiales entre los que destacan los Sacbs.

En la zona de estudio, los Sacbs constituyen el sustento predominante dentro del sector agrícola. Para al año 2017 esta actividad productiva cubría $18.86 \%$ de la región, lo que lo convierte en un eslabón, no solo en el aspecto social fuentes de empleo-, sino como ecosistema crucial en la conservación de la biodiversidad y lo servicios ecosistémicos (Moguel y Toledo, 1999; Williams Linera et al., 2002; Pineda-López et al., 2005; Redo, Bass y Millington, 2009; Perfecto y Vandeermer, 2010).

Como en otros casos, los ecosistemas naturales muestran una tendencia a disminuir su superficie (Lambin y Meyfroidt, 2010), por cambios de uso del suelo con vocación forestal a tierras de cultivo y pastoreo para el desarrollo de actividades agropecuarias, e incluso para la apropiación y acaparamiento de tierras (Paz y Latam, 2019).

Los patrones de cambio observados en los Sacbs de los 13 municipios veracruzanos que conforman el área de análisis están fuertemente condicionados por las actividades humanas que están modificado el funcionamiento de los ecosistemas naturales (bosque mesófilo de montaña y bosque tropical caducifolio, entre otros), y por ende la capacidad para producir y proveer servicios ecosistémicos (Mas y Fernández, 2003). A lo anterior, se suma la presión generada por la ampliación de la mancha urbana (Ambinakudige y Choi, 2009; Bass, 2006; Ellis et al., 2010; Schmitt-Harsh, 2013) y la consecuente sobre demanda de servicios y bienes ambientales.

El estudio de la magnitud de las transformaciones de los Sacbs muestra concordancia entre el análisis cuantitativo y cualitativo realizado; los cambios en el paisaje agroforestal están determinados por la situación y contexto socioambiental y político en la escala temporal y se reflejan en la configuración del territorio y las actividades que se desarrollan en el mismo a escala espacial.

A pesar de que para el período de estudio (1993 2017) se registró un aumento en la superficie de Sacbs, en las etapas intermedias las coberturas del Sacbs que se transformaron se convirtieron en su mayoría en acahuales debido al abandono de esta actividad. Entre las causas identificadas de 1993 a 2003, destacan la baja productividad (tanto en calidad como en cantidad) debido por la presencia de plagas y enfermedades (presencia de roya en los años de 1989, 1998 y 2015) (FIRA, 2016), que sumadas al bajo valor del grano aromático en el mercado nacional e internacional (OIC, 2011), y la desaparición tanto de instituciones como de apoyos estratégicos que fueron fundamentales para el desarrollo y mantenimiento de este sector en México.

Entre las dependencias gubernamentales que fueron clave para el desarrollo de este sector se destacaron el Inmecafe, el Consejo Mexicano del Café, la Alianza para el campo (Durante 1996) y el Procampo de la Sagarpa, así como el Pronare (ambas iniciaron operaciones en el año de 1993), esta última de la Comisión Nacional Forestal (Conafor). A pesar de los esfuerzos, estos no fueron suficientes para apuntalar esta actividad productiva en la zona, debido a la falta de acompañamiento en los procesos relacionados con los apoyos, lo que promovió aún más el fenómeno de emigración histórico que se presenta en la región al desaparecer las fuentes de ingreso relacionadas con la actividad. Esta situación, a su vez, provoco una disminución en la disponibilidad de la mano de obra para la cafeticultura en esta zona. Por lo anterior en el periodo correspondiente de 1993 a 2003, la tasa de cambio de los Sacbs fue negativa $(-0.95 \%)$, situación que coincide con eventos como el de 2002, en el que se detecta una baja productividad y bajos precios, por lo que los entrevistados manifiestan que desde 1998 han desaparecido muchas fincas en la parte alta de la cuenca La Antigua.

Por otra parte, durante el periodo 2003 a 2017 los Sacbs tuvieron un repunte en su superficie, relacionado con la recuperación de fincas abandonadas, algunas de ellas con más de diez años de abandono que fueron habilitadas para la producción del llamado "Café orgánico" lo que les da un valor agregado a los productos agropecuarios con estas características (Expansión, 2019). Dado que la preferencia por los llamados "productos orgánicos" va en aumento a partir del año 2000 no sólo en el país sino también a nivel internacional; los productores se ven motivados a mantener los Sacbs bajo ese concepto respetando las condicionantes 
que les obliga la certificación; que en la mayoría de los casos es debido a la convicción personal de no usar agroquímicos y pesticidas por cuestiones de salud, no obstante que, en algunos casos, implica costos mayores en insumos y mano de obra. En este periodo, desaparecen los apoyos del Consejo Mexicano del Café, Alianza para el Campo y otros del gobierno veracruzano dedicados a este sector. Sin embargo, el repunte del precio y el fomento al consumo del café mexicano (a pesar de los volúmenes importados) genera una situación favorable para los productores.

Es importante destacar que los entrevistados señalan que las actividades agrícolas (diferentes al cultivo de café) han disminuido desde el año 2000 en la cuenca. Tal es el caso del cultivo comercial del maíz que, al no ser competitivo en el mercado con las importaciones de maíz, se ha reducido a un cultivo de subsistencia o ha sido sustituido por cultivos de papa, haba y frijol en las regiones superiores a los $2500 \mathrm{~m} \mathrm{snm}$; mientras que debajo de esa cota se han extendido los cultivos de chayote, cítricos y caña de azúcar.

En relación con el uso de suelo "infraestructura" crecimiento de los asentamientos humanos- se desarrolla de forma desordenada y voraz desde inicios de 1990, con una tasa de crecimiento de $3.48 \%$ en 24 años, pasando de 194.01 ha en el año 1993 a 440.68 ha en el año 2017. Lo anterior refleja la demanda de más espacios, servicios, vías de comunicación y otros que consecuentemente modifican los paisajes tanto naturales como agropecuarios (EspinozaGuzmán, 2012; Reyes, 2015; Reyes, 2018).

\section{CONCLUSIONES}

El estudio permitió identificar las trasformaciones de los sistemas agroforestales cafetaleros bajo sombra en la parte alta de la cuenca de La Antigua durante el periodo analizado, a partir de lo cual es posible reconocer el efecto de los diferentes factores relacionados con actividades humanas, tales como el crecimiento de la mancha urbana, en los procesos de cambios de uso del suelo. Por otro lado, cabe señalar que de 1993 a 2017 los Sacbs se ha mantenido sin cambio $48.97 \%$ en la zona de estudio, y que aun cuando se trata de una actividad agrícola, se mantiene en armonía con el bosque mesófilo de montaña como lo indican diversos autores en otros estudios.

Desde el enfoque metodológico usado, es importante señalar que la teledetección para el análisis espaciotemporal de cambios de cobertura y uso del suelo permite dar seguimiento a las modificaciones superficiales de forma efectiva, rápida y confiable. Por otro lado, el análisis de contenido de las entrevistas de profundidad, permitieron reconocer los elementos que influyeron en el cambio de uso de suelo, condicionados por las actividades humanas, y que coincidió con la valoración cuantitativa del análisis espaciotemporal de los cambios de uso del suelo.

Los resultados de este estudio constituyen un insumo de utilidad para apoyar los diversos esfuerzos en la toma de decisión vinculadas con el ordenamiento territorial, la administración de recursos naturales, la identificación de zonas prioritarias para la conservación, el manejo forestal, zonas vulnerables ante el cambio climático, entre otros. Es necesario trabajar en la definición de líneas de acción específicas para la porción alta de la cuenca La Antigua, con una visión de manejo integrado de paisaje que considere su papel como parte del Corredor Ecológico de la Sierra Madre Oriental.

\section{REFERENCIAS}

Agarwal, C., Green, G. M., Grove, J. M., Evans, T. P., \& Schweik, C.M. (2002). A review and assessment of land-use change models: Dynamics of space, time, and human choice. Burlington, VT: United States Department of Agriculture, Forest Service, Northeastern Research Station.

Alexander, P., Rounsevell, M. D. A., Dislich, C., Dodson, J. R., Engström, K., \& Moran, D. (2015). Drivers for global agricultural land use change: The nexus of diet, population, yield and bioenergy. Global Environmental Change, 35, 138-147. doi:10.1016/j.gloenvcha.2015.08.011

Ambinakudige, S., \& Choi, J. (2009). Global coffee marketing fluence on land use and land cover change in the Western Ghats of India. Land Degradation \& Development, 20(3), 327-335. doi:10.1002/ldr.921

Archer D. W., Dawson, J, Kreuter, U. P., Hendrickson, M., \& Halloran J. M. (2008). Social and political influences on agricultural systems. Renewable Agriculture and Food Systems, 23(4), 272-284. doi:10.1017/S174217050700169X 
Argañaraz, J. P., \& Entraigas, I. (2011). Análisis comparativo entre las máquinas de vectores

soporte y el clasificador de máxima probabilidad para la discriminación de cubiertas de suelo. Asociación Española de Teledetección. Revista de Teledetección, 36, 26-39.

Bardin, L. (1996). El análisis de contenido. Madrid: Ediciones Akal.

Bass, J. O. J. (2006). Forty years and more trees: land cover change and coffee production in Honduras. Southeastern Geographer, 46(1), 5165. doi:10.1353/sgo.2006.0002

Bosselmann, A. E. (2012). Mediating factors of land use change among coffee farmers in a biological corridor. Ecological Economics, 80, 7988. doi:10.1016/j.ecolecon.2012.05.007

Castillo-Capitán, G., Ávila-Bello, C. H., López-Mata, L., \& De León, G. F. (2014). Structure and tree diversity in traditional Popoluca coffee agroecosystems in the Los Tuxtlas Biosphere Reserve, Mexico. Interciencia, 39(9), 608-619.

Cerda, L. J. \& Villarroel, Del P. L. (2008). Evaluación de la concordancia inter-observador en investigación pediátrica: Coeficiente de Kappa. Revista Chilena Pediatría, 79(1), 54-58. doi:10.4067/S037041062008000100008

Chomitz, K. M. \& Gray, D. A. (1996). Roads, land use, and deforestation: a spatial model applied to Belize. The World Bank Economic Review, 10(3), 487-512.

Chuvieco, S. E. (2008). Teledetección Ambiental. La observación de la Tierra desde el Espacio. España: Ariel Ciencia.

Ellis. E. A., Baerenklau, K. A., Marcos-Martínez, R. \& Chávez, R. (2010). Land use/land cover dynamics and drivers in a low-grade marginal coffee growing region of Veracruz, Mexico. Agroforestry Systems, 80(1), 61-84. doi:10.1007/s10457-010-9339-2

Espinoza-Guzmán, M. A. (2012). La transformación de los paisajes en la parte alta de la cuenca del río la Antigua. Un análisis desde la perspectiva constructivista de los Sistemas Complejos, Tesis doctoral, Universidad Veracruzana, Centro de Investigaciones Tropicales. Xalapa, Veracruz, México.

Expansión. (2019). México es el cuarto productor de alimentos orgánicos en el mundo. Revista Expansión. Recuperado de: https://expansion.mx/economia/2018/12/27/mexico-es-elcuarto-productor-de-alimentos-organicos-en-el-mundo

Food and Agriculture Organization [FAO] (1996). Forest resources assessment 1990. Survey of tropical forest cover and study of change processes. (Technical report of a major global cooperative effort coordinated by the Forest Resources Assessment 1990 Project). Rome: Food and Agriculture Organization.
Fideicomiso Instituidos en Relación con la Agricultura [FIRA] (2016). Panorama agroalimentario. Café 2016. Dirección de investigación y evaluación económica y sectorial. Recuperado de: https://www.fira.gob.mx/InvYEvalEcon/EvaluacionIF

Foley, J. A., R. De Fries, G. P. Asner, C. Barford, G. Bonan, S. R. Carpenter, F. S. Chapin, III, M. T. Coe, G. C. Daily, H. K. Gibbs, J. H. Helkowski, T. Holloway, E. A. Howard, C. J. Kucharik, C. Monfreda, J. A. Patz, I. C. Prentice, N. Ramankutty, \& P. K. Snyder. (2005). Global consequences of land use. Science, 309(5734), 570-574. doi:10.1126/science.1111772

Fonseca-Cepeda, V., Idrobo, C. J., \& Restrepo, S. (2019). The changing chagras: traditional ecological knowledge transformations in the Colombian Amazon. Ecology and Society, 24(1), 8. doi:10.5751/ES10416-240108

García, S., B., Gallardo, L. F., Nava, T., M. E., Ruiz, R. O., \& Escamilla, P. E. (2006). Factores que limitan la certificación de café orgánico en el esquema de comercio justo en cinco organizaciones de México. Revista Mexicana del Caribe, 10(19), 205-226.

Geist, H. J., \& Lambin, E. F. (2001). What Drives Tropical Deforestation? A meta-analysis of proximate and underlying causes of deforestation based on sub-national case study evidence. LUCC Report Series, 4, International Project Office, Belgium, 116.

Hoffmann, O. (1993). Rumbos y Paisajes de Xico. Geografía de un municipio de la sierra de Veracruz: México: L'Institut Français de Recherche pour le Développement en Coopération - Instituto de Ecología, A. C.

Instituto Nacional de Estadística, Geografía e Informática [Inegi] (2010). Áreas geoestadísticas municipales. Marco Geoestadístico Nacional V.6.

Instituto Nacional de Estadística, Geografía e Informática [Inegi] (2005). Modelos Digitales de Elevación. Recuperado de: http://mapserver.inegi.gob.mx/geografia/espanol/normativida $\mathrm{d} / \mathrm{mde} / \mathrm{menu} \cdot \mathrm{cfm}$ ?c $=198$.

Jenness, J., \& Judson, J. W. (2007). Cohen's Kappa and Classification Table Metrics 2.1a: An ArcView $3 x$ Extension for Accuracy Assessment of Spatially-Explicit Models, EE. UU,

Lambin, E. F., Geist, H. J., \& Lepers, E. (2003). Dynamics of land use and land cover change in tropical regions. Annual review environment resources, 28(1), 205-241. doi: 10.1146/annurev.energy.28.050302.105459

Lambin, E. F., Turner, B. L., Helmut. J. G., Agbola. S. B., Angelsen, A., Bruce, J. W., Coomes, O. T., Dirzo, R., Fischer. G., Folke, C., George P. S., Homewood, K., Imbernon, J., Leemans, R., Li, X., Moran E. F., Mortimore, M., Ramakrishnan, P. S., Richards J. F., Skanes, H., Steffen, W., Stone, G. D., Svedin, U., Veldkamp, T. A., Vogel, C., \& Xu, J. (2001). The causes of land-use and land- 
cover change: moving beyond the myths. Global Environmental Change, 11(4), 261-269. doi:10.1016/S0959-3780(01)00007-3

Lambin, E. F., \& Meyfroidt, P. (2010). Land use transitions: Socioecological feedback versus socio-economic change. Land Use Policy, 27(2), 108-118. doi: 10.1016/j.landusepol.2009.09.003

Lofland, J., \& Lofland, L. H. (eds) (1995). Analyzing Social Settings: a guide to qualitative observation and analysis. Belmont, CA: Wadsworth Publishing Company.

Mahoney, C. (1997). Common qualitative meths. En Joy Frechtling and Laure Sharp Inc. (Ed.), User-friendly bandbook for mixed methods evaluations. National Science Foundation.

Mas, J. F. (1999). Monitoring land-cover changes: a comparison of change detection techniques. International Journal of Remote Sensing, 20(1), 139-152. doi: 10.1080/014311699213659

Mas, J. F., \& Fernández, T. (2003). Una evaluación cuantitativa de los errores en el monitoreo de los cambios de cobertura por comparación de mapas. Investigaciones Geográficas, 51, 73-87.

Moguel P., \& Toledo M. V. M., (1999). Biodiversity conservation in traditional coffee systems of Mexico. Conservation Biology, 13(1), 11-21. doi:10.1046/j.1523-1739.1999.97153.x

Muñoz-Villers, L. \& López-Blanco, J. (2007). Land use/cover changes using Landsat TM/ETM images in a tropical biodiverse mountainous area of central-eastern Mexico. International Journal of Remote Sensing, 29(1), 71-93. doi:10.1080/01431160701280967

Organización Internacional del Café [OIC]. (2011). Precios del café. Recuperado de: http://www.ico.org/ES/coffee_pricesc.asp?section=Estad $\%$ E Dstica

Palacio-Prieto, J., Sánchez-Salazar, M., Casado-Izquierdo, J., PropinFrejomil, E., Delgado-Campos, J., Velázquez-Montes, A., ChiasBecerril, L., Ortiz-Álvarez, M., González-Sánchez, J., NegreteFernández, G., Gabriel-Morales, J., Márquz-Huitzil, R., NiedaManzano, T., Jiménez-Rosenberg, R., Muñoz-López, E., Ocaña Nava, D., Juárez Aguirre, E., Anzaldo Gómez, C., Hernández Esquivel, J., Valderrama-Campos, K., Rodríguez-Carranza, J., Campos-Campuzano, J., Vera Llamas-Cruz, H., \& CamachoRamírez, C. (2004). Indicadores para la caracterización y ordenamiento de territorio. México: Semarnat - INE, UNAM, IG, Sedesol.

Paz, C. A., \& Latam, M. (2019). Día de la Tierra: deforestación hace que Latinoamérica sea más vulnerable al cambio climático. Recuperado de: https://sostenibilidad.semana.com/impacto/articulo/dia-de-latierra-deforestacion-hace-que-latinoamerica-sea-mas-vulnerableal-cambio-climatico/43896
Perfecto, I. \& Vandermeer, J. (2010). The agroecological matrix as an alternative to the land-sparing / agriculture intensification model. Proceedings of the National Academy of Sciences, 107(13), 5786-5791. doi:10.1073/pnas.0905455107

Pineda-López, M. R., Ortíz-Ceballos, G., \& Sánchez-Velásquez, L. R. (2005). Los cafetales y su papel en la captura de carbono: un servicio ambiental aún no valorado en Veracruz. Madera y Bosques, 11(2), 3-14. doi: 10.21829/myb.2005.1121253

Piñar-Álvarez, M.A., Espinoza-Guzmán, M.A., Jiménez-Olivencia, Y., \& Loreto-Bermúdez, F.D. (2016). En Márquez-Domínguez, J. A., Jurado-Almonte, J. M., \& Pazos-García, F. J. (Coords.), Desarrollo local en territorios de fronteras (pp. 169-186). Huelva, España: Universidad de Huelva, Servicio de publicaciones, Collectanea, $\mathrm{n}^{\circ}$ 205.

Ponette-González, A. G. (2007). 2001: A household analysis of Huastec Maya agriculture and land use at the height of the coffee crisis. Human Ecology, 35(3), 289-301. doi: 10.1007/s10745-006-9091-4

Redo, D., Bass, J. J., \& Millington, A. C. (2009). Forest dynamics and the importance of place in western Honduras. Applied Geography, 29(1), 91-110. doi: 10.1016/j.apgeog.2008.07.007

Reyes, G. Z. F. (2015). Dinámica del cambio de cobertura y uso de suelo en la porción alta de la cuenca del rio la Antigua (Años 1993, 1999, 2010 Y 2015). Tesis de licenciatura. , Universidad Veracruzana, Facultad de Ciencias Químicas. Xalapa, Veracruz.

Reyes G., F. Z. (2018). Influencia del cambio de cobertura y uso del suelo en el volumen medio anual de escurrimiento natural (años 1993, 2003 y 2017), en la parte alta de la cuenca del río La Antigua, Veracruz, México. Tesis de maestría. Instituto Politécnico Nacional, Centro Interdisciplinario de Investigaciones sobre Medio Ambiente y de Desarrollo.

Schmitt-Harsh, M. (2013). Landscape change in Guatemala: Driving forces of forest and coffee agroforest expansion and contraction from 1990 to 2010. Applied Geography, 40, 40-50. doi:10.1016/j.apgeog.2013.01.007

Touraine, A. (1997). ¿Podemos vivir juntos? La discusión pendiente: El destino del hombre en la aldea global. Buenos Aires: FCE de Argentina.

Turner, B. L., Lambin, E. F., \& Reenberg, A. (2007). The emergence of land change science for global environmental change and sustainability. Proceedings of the National Academy of Sciences, 104(52), 20666-20671. doi: 10.1073/pnas.0704119104

Velázquez A., Mas, J. F., Gallegos J., R. D., Mayorga-Saucedo, R., Alcántara P., C., Castro, R., \& Palacio, J. L. (2002). Patrones y 
tasas de cambio de uso del suelo en México. Gaceta ecológica, 62, $21-37$.

Verburg P., Paul, H., Schot, P., Dijst, M. J., \& Veldkamp, A. (2004). Land use change modelling: current practice and research priorities. GeoJournal, 61(4), 309-324. doi: 10.1007/s10708-004-4946-y
Williams-Linera, G., Manson, R. H., \& Isunza-Vera, E. (2002). La fragmentación del bosque mesófilo de montaña y patrones de uso de suelo en la región oeste de Xalapa, Veracruz, México. Maderay Bosques, 8(1), 73-89. doi: 10.21829/myb.2002.811307

ANEXO 1

Preguntas para entrevista semiestructurada

1.- ¿Cuáles son las regiones, paisajes o parajes que reconoce en su municipio?

2.- Indique cuáles ¿Cuáles son las actividades productivas que identifica en estas áreas?

¿Desde su punto de vista ha observado cambios en el paisaje, entorno?

3.- ¿Ildentifica en el tiempo algún cambio en las actividades productivas -en cuáles-?

4.- ¿En qué año se generaron estos cambios?

5.- ¿Cuáles considera que fueron las causas que dieron pauta a estos cambios (personas, instituciones, dependencias, programas, planes, etc.)?

6.- ¿Como resultado del cambio en el paisaje, identifica algún problema ambiental (agua, suelo, aire, bosque, vida silvestre, cultivos, turismo, basura, etc.)?

7.- ¿Como resultado del cambio en el paisaje, identifica algunos problemas sociales y económicos (salud, marginación, desempleo, migración, inseguridad, etc.)?

8.- ¿Cuáles han sido las consecuencias, efectos, repercusiones (paisaje, personas, instituciones, dependencias, programas, etc.)?

9.- ¿En quienes ha repercutido dichas modificaciones, como producto de las alteraciones en el paisaje?

10.- ¿Qué es lo que se está haciendo para minimizar los efectos?

11.- ¿Qué acciones propondría para minimizar estos efectos (paisaje, personas, instituciones, dependencias, programas, etc.)?

12.- ¿Cómo se espera sean en el futuro los paisajes, parajes o zonas?

Los cuestionamientos anteriores sirvieron como base para los diálogos con los actores clave identificados en la región (al menos dos entrevistas por municipalidad), quienes conocen y reconocen hechos históricos que han marcado la parte alta de la Cuenca de La Antigua, aportando elementos de análisis y discusión sobre los aspectos económico, ecológico y social referente a la transformación de los paisajes.

Manuscrito recibido el 7 de junio de 2019

Aceptado el 19 de noviembre de 2019

Publicado el 24 de abril de 2020

Este documento se debe citar como:

Espinoza-Guzmán, M. A., Sánchez V., L. R., Pineda L., M. R., Sahagún S., F. J., David Aragones B., D., \& Reyes G., Z. F. (2020).
Dinámica de cambios en el agroecosistema de cafetal bajo sombra en la cuenca alta de La Antigua, Veracruz. Madera y Bosques, 26(2), e2621974. doi: 10.21829/myb.2020.2621974 